\title{
Melanoma Imaging with Highly Specific PET Probes: Ready for Prime Time?
}

$\mathbf{T}$ he incidence of cutaneous melanoma has shown an increase over the last 3 decades, with most of the rise due to thin rather than thick tumors (1). Melanoma accounts for up to $90 \%$ of all deaths caused by skin cancer and has a high metastatic potential if the primary lesion shows any adverse features such as thickness of more than $1 \mathrm{~mm}$, ulceration, increased mitotic activity, or invasion through the dermis (Clark level IV or V) (2). According to the National Cancer Institute, in 2010 the estimated number of new cases and deaths from melanoma in the United States was 68,130 and 8,700, respectively (3).

See page 115

The most important prognostic factor of newly diagnosed melanomas is regional lymph node status. Although distant metastases are relatively uncommon at the time of diagnosis, approximately $20 \%$ of melanoma patients harbor metastatic involvement in the regional nodal basin. This metastatic involvement is currently diagnosed with high accuracy using sentinel node biopsy (SNB) followed by, in the case of positive findings, completion lymph node dissection (CLND) (4). Although still under debate, it is suggested that SNB-guided CLND

Received Jul. 21, 2010; revision accepted Aug. 11, 2010.

For correspondence or reprints contact: Heikki Minn, Department of Oncology and Radiotherapy, Turku PET Centre, Turku University Hospital, P.O. Box 52, 20521 Turku, Finland.

E-mail: heminn@utu.fi

COPYRIGHT (C 2011 by the Society of Nuclear Medicine, Inc.

DOI: 10.2967/jnumed.110.080523 confers a better prognosis than therapeutic lymph node dissection performed for patients with clinically evident metastases (5).

\section{CURRENT PRACTICE IN STAGING OF MELANOMA WITH PET}

Guidelines for the American Joint Committee on Cancer staging system of melanoma were recently published; however, these guidelines continually evolve (6). Although the importance of radiographic and nuclear medicine techniques for staging is recognized, the appropriate and timely use of these techniques both for the initial evaluation and for the subsequent follow-up are not well defined. Although CT and MRI are the preferred methods for assessing lung nodules and brain metastases, respectively, there is good evidence for using PET/CT in detecting metastases in the deep soft tissue, lymph nodes, and viscera (7). The clinical data on PET and PET/CT are based solely on ${ }^{18} \mathrm{~F}$ FDG imaging, which is expected to change management in $15 \%-64 \%$ of cases when melanoma patients with American Joint Committee on Cancer stage III-IV are evaluated after conventional diagnostic work-up (7). However, the sensitivity of ${ }^{18} \mathrm{~F}-\mathrm{FDG}$ PET is not sufficient in the initial diagnosis of metastasis in regional lymph nodes, for which SNB preceded by lymphoscintigraphy is the gold standard $(8,9)$. Given the sometimes small burden of metastatic cells, the insensitivity of a general metabolic agent such as ${ }^{18} \mathrm{~F}-\mathrm{FDG}$ is hardly surprising because its tumor-to-background ratio remains below the resolution of the PET detection system in imaging the clinically uninvolved sentinel node basin.

\section{NOVEL TRACERS FOR IMAGING OF MELANOMA METASTASES}

As a consequence of the limited benefit of ${ }^{18} \mathrm{~F}$-FDG in evaluating sentinel nodes, the desire to successfully combat recurrent and metastatic melanoma has sparked interest in the development of tracers relating to specific characteristics of melanoma cells (1012). A class of compounds that depicts melanin biosynthesis and metallopeptide binding to melanocortin type 1 receptors overexpressed in melanomas belongs to this category of novel and promising agents, and antibody and angiogenesis imaging continue to reside in the arena of research and development. Initial experience in $\gamma$-emitting radioisotopes has gradually shifted to interest in labeling melanoma probes with positron-emitting isotopes, for which ${ }^{18} \mathrm{~F}$ is the natural substitute of iodinated analogs. This shift is due to the requirement of high sensitivity, especially if the completion of SNB is the focus, but also reflects the present increase in clinical PET/CT facilities. Recently, the preclinical targeting of B16F10 murine melanoma-expressing melanocortin type 1 receptor with a rhenium-cyclized metallopeptide $\left({ }^{18} \mathrm{~F}-\mathrm{FB}\right.$ RMSH-1) was successfully achieved (13). The publication of that paper was followed by 2 studies on the imaging of the B16F10 model using the melanin-affine benzamide analog $N$-[2-(diethylamino) ethyl]-4- ${ }^{18}$ F-fluorobenzamide $\left({ }^{18} \mathrm{~F}-\mathrm{FBZA}\right)$ (14) and fluoronicotinamide analog ${ }^{18} \mathrm{~F}$-6-fluoro- $N$-[2-(diethylamino)ethyl] pyridine-3-carboxamide $\left({ }^{18} \mathrm{~F}-\mathrm{MEL} 050\right)$ (15). None of the 3 tracers showed avidity to xenografted amelanotic A375M tumors. The uptake of ${ }^{18} \mathrm{~F}-\mathrm{FBZA}$ in $\mathrm{B} 16 \mathrm{~F} 10$ was comparable to that of ${ }^{18} \mathrm{~F}-$ FDG, and the uptake of ${ }^{18} \mathrm{~F}-\mathrm{MEL} 050$ in B16F0 was higher than that of ${ }^{18} \mathrm{~F}-\mathrm{FDG}$ (13-15). Although direct comparison 
between the 3 melanoma probes may not be pertinent, it is obvious that the tumor-to-background ratio is higher using the melanin-targeting compounds in the B16 melanoma model, making the latter 2 compounds superior at detecting small-volume melanoma deposits. The differences in biodistribution and general pharmacokinetic properties among the 3 tracers are interesting and need further investigation in the setting of therapeutic interventions in other mouse melanoma models and, ultimately, if development programs continue to be given the green light, in melanoma patients. Both ${ }^{18} \mathrm{~F}-\mathrm{FBZA}$ and ${ }^{18} \mathrm{~F}-\mathrm{MEL} 050$ are capable of imaging experimental lung metastases, and tracer uptake seems to reflect metastatic burden $(14,15)$. In subcutaneous $\mathrm{B} 16 \mathrm{~F} 0$ grafts, the 9-fold-greater tumorto-background ratio of ${ }^{18} \mathrm{~F}-\mathrm{MEL} 050$ than ${ }^{18} \mathrm{~F}-\mathrm{FDG}$ at $2 \mathrm{~h}$ is promising and suggests the high diagnostic potential of melanin-targeted imaging in clinical oncology (15).

\section{DETECTION OF REGIONAL LYMPH NODES WITH ${ }^{18} \mathrm{~F}$-MEL050}

In the current issue of The Journal of Nuclear Medicine, the Australian group presents a perilesional approach of tracer administration that is applied to the detection of regional lymph node metastases after the demonstration of the rapid uptake and high retention of ${ }^{18} \mathrm{~F}$ MEL050 in B16F0 melanoma with specificity for melanin (16). The authors implanted in the $\mathrm{C} 57 \mathrm{BL} / 6$ mouse dorsal foot B16-BL/6 metastatic melanoma cells carrying a copy of the mCherry transgene and with a propensity to metastasize in the popliteal lymph node (PLN). After PLN metastasis developed in 9 of 49 mice, ${ }^{18} \mathrm{~F}-\mathrm{MEL} 050$ was injected either perilesionally or intravenously with a dose that was 15 -fold higher in the systemic than in the local route. The tumor load in PLNs was assessed with high-resolution animal ultrasound showing morphologic changes suggestive of nodal involvement and by quantitative PCR assaying the mCherry transgene of the resected nodal tissue. The findings of Denoyer et al. (16) indicate superior melanoma-spe- cific tracer accumulation in metastatic PLNs when the local route was used, with sensitivities of $100 \%$ and $60 \%$ and node-to-background ratios of 48 and 6.8 for local and systemic administrations, respectively. Both injection routes were able to detect lung metastases commonly seen in this tumor model. Importantly, there were no false-positives using either means of tracer administration, and in line with this, the perilesional route did not indicate any unspecific trapping of tracer in draining nodes. The authors concluded that ${ }^{18} \mathrm{~F}-\mathrm{MEL} 050$ may allow sensitive and specific identification of regional lymph node metastasis-a conclusion clearly substantiated by the presented data.

\section{POTENTIAL CLINICAL IMPLICA- TIONS FOR ${ }^{18} \mathrm{~F}$-MEL050 IMAGING}

The study of Denoyer et al. (16) is an example of translational research with great clinical potential, and the application of ${ }^{18}$ F-MEL050 imaging in melanoma patients is easy to imagine. In logical continuum to their previous study (15), this highly specific melanoma probe- ${ }^{18} \mathrm{~F}$ MEL050 - has passed the preclinical validation tests, suggesting that it images tumor burden better and likely detects smaller metastases than does ${ }^{18} \mathrm{~F}-\mathrm{FDG}$. This feature of ${ }^{18} \mathrm{~F}$-MEL050 would aid surgery and focused radiotherapy, which, in the case of melanoma, may best be delivered with conformal beams and higher-than-standard daily fractions. Improved metastatic staging disclosing occult lesions in the lungs and brain would help in the overall choice between different treatment modalities and in monitoring the effects of immunomodulatory and molecular therapy, for which it is hoped that less inflammation-associated tracer uptake, compared with ${ }^{18} \mathrm{~F}-\mathrm{FDG}$ uptake, would be observed. In patients who present with melanoma metastases and no known primary site, it would be enticing to use ${ }^{18} \mathrm{~F}-\mathrm{MEL} 050$ instead of ${ }^{18} \mathrm{~F}$ FDG, which does not perform well in this special diagnostic challenge (17). Whether ${ }^{18} \mathrm{~F}-\mathrm{MEL} 050$ holds promise in detecting metastatic involvement in the draining lymph nodes perhaps below the classic 5- to 6-mm resolution limit typical of
${ }^{18}$ F-FDG (which generally suffers from inability to image micrometastases of multiple tumor types) remains to be seen. In contrast, concurrent perilesional and systemic injection of ${ }^{18} \mathrm{~F}-$ MEL050 might become the first imaging test preceding SNB and replacing most conventional imaging studies. Patients with a positive regional ${ }^{18} \mathrm{~F}-\mathrm{MEL} 050 \mathrm{PET} / \mathrm{CT}$ result would immediately proceed to CLND and avoid SNB, provided that distant metastases were not detected on whole-body acquisition. A 1-stop shop for diagnostic work-up of melanoma staging would become possible with ${ }^{18}$ F-MEL050, bearing in mind that patients with negative scan results would continue to require SNB and low-risk primary cases would not benefit from imaging (6).

\section{SUGGESTIONS FOR the FUTURE}

Before all this is reality and ${ }^{18} \mathrm{~F}-$ MEL050, or another melanoma imaging molecular probe, is available for clinical PET, additional and well-designed studies are in order. A prospective and likely multicenter imaging protocol comparing the novel melanoma probe against ${ }^{18} \mathrm{~F}-\mathrm{FDG}$ needs to be developed. It would be important to test the tumor burden concept also clinically, especially when introducing the new agent for imaging regional lymph nodes together with SNB. Association of tracer uptake with mitotic activity, serum lactate dehydrogenase level, and all previously established prognostic factors is of importance as well (18). We foresee that this clinical development will happen soon. Many other recent success stories of the use of non- ${ }^{18} \mathrm{~F}-\mathrm{FDG}$ tracers in the nuclear medicine and oncology communities make the use of ${ }^{18} \mathrm{~F}$ MEL050 in the case of melanoma imaging encouraging. We congratulate the authors for the current novel scientific findings $(15,16)$, which we hope will offer a cure for an increasing number of patients afflicted by this devastating disease.

\section{Heikki Minn \\ Pia Vihinen \\ Turku University Hospital \\ Turku, Finland}




\section{REFERENCES}

1. Bataille V, de Vries E. Melanoma-part 1: epidemiology, risk factors, and prevention. BMJ. 2008;337: a2249.

2. Garbe C, Peris K, Hauschild A, et al. Diagnosis and treatment of melanoma: European consensus-based interdisciplinary guideline. Eur J Cancer. 2010;46: 270-283.

3. National Cancer Institute. Melanoma. Available at: www.cancer.gov/cancertopics/types/melanoma. Accessed November 17, 2010.

4. van Akkooi AC, Voit CA, Verhoef C, Eggermont AMM. New developments in sentinel node staging in melanoma: controversies and alternatives. Curr Opin Oncol. 2010;22:169-177.

5. Pasquali S, Mocellin S, Campana LG, et al. Early (sentinel lymph node biopsy-guided) versus delayed lymphadenectomy in melanoma patients with lymph node metastases: personal experience and literature meta-analysis. Cancer. 2010;116:1201-1209.

6. Mohr P, Eggermont AMM, Hauschild A, Buzald A. Staging of cutaneous melanoma. Ann Oncol. 2009; 20(suppl 6):vi14-vi21.

7. Krug B, Crott R, Lonneux M, Baurain JF, Pirson AS, Vander Borght T. Role of PET in the initial staging of cutaneous malignant melanoma: systematic review. Radiology. 2008;249: 836-844.

8. Jiménez-Requena F, Delgado-Bolton RC, FernándezPérez C, et al. Meta-analysis of the performance of ${ }^{18} \mathrm{~F}$-FDG PET in cutaneous melanoma. Eur $\mathrm{J}$ Nucl Med Mol Imaging. 2010;37:284-300.

9. El-Maraghi RH, Kielar AZ. PET vs sentinel lymph node biopsy for staging melanoma: a patient intervention, comparison, outcome analysis. J Am Coll Radiol. 2008;5:924-931.

10. Cheng Z, Zhang L, Graves E, et al. Small-animal PET of melanocortin 1 receptor expression using a ${ }^{18} \mathrm{~F}$-labeled $\alpha$-melanocyte-stimulating hormone analog. J Nucl Med. 2007;48:987-994.

11. Greguric I, Taylor SR, Denoyer D, et al. Discovery of $\left[{ }^{18} \mathrm{~F}\right] \mathrm{N}$-(2-(diethylamino)ethyl)-6fluoronicotinamide: a melanoma positron emission tomography imaging radiotracer with high tumor to body contrast ratio and rapid renal clearance. J Med Chem. 2009;52:5299-5302.

12. Decristoforo C, Hernandez Gonzalez I, Carlsen J, et al. ${ }^{68} \mathrm{Ga}$ - and ${ }^{111} \mathrm{In}$-labelled DOTA-RGD peptides for imaging of $\alpha_{\mathrm{v}} \beta_{3}$ integrin expression. Eur $J$ Nucl Med Mol Imaging. 2008;35:1507-1515.
13. Ren G, Liu Z, Miao Z, et al. PET of malignant melanoma using ${ }^{18} \mathrm{~F}$-labeled metallopeptides. $J$ Nucl Med. 2009;50:1865-1872.

14. Ren G, Miao Z, Hongguang L, et al. Melanintargeted preclinical PET imaging of melanoma metastasis. J Nucl Med. 2009;50:1692-1699.

15. Denoyer D, Greguric I, Roselt P, et al. High-contrast PET of melanoma using ${ }^{18} \mathrm{~F}-\mathrm{MEL} 050$, a selective probe for melanin with predominantly renal clearance. J Nucl Med. 2010;51:441-447.

16. Denoyer D, Potdevin T, Roselt P, et al Improved detection of regional melanoma metastasis using 18F-6-fluoro- $N$-[2-(diethylamino)ethyl] pyridine3-carboxamide, a melanin-specific PET probe, by perilesional administration. J Nucl Med. 2010;52: 115-122.

17. Kamposioras K, Pentheroudakis G, Pectasides D, Pavlidis N. Malignant melanoma of unknown primary site: to make the long story short-a systematic review of the literature. Crit Rev Oncol Hematol. May 28, 2010 [Epub ahead of print].

18. Balch CM, Gershenwald JE, Soong S-j, et al. Final version of 2009 AJCC melanoma staging and classification. J Clin Oncol. 2009;27:61996206. 